\title{
Load distribution and skid compensation control of omni-directional vehicle with independent driving modules
}

\author{
Yuji ISHII* and Kazuo NAKAZAWA* \\ *Faculty of Science and Technology, Keio University, \\ 3-14-1 Hiyoshi, Kohoku-ku, Yokohama, 223-8522 Japan \\ E-mail: nakazawa@sd.keio.ac.jp
}

Received: 4 April 2016; Revised: 8 August 2016; Accepted: 4 December 2016

\begin{abstract}
The demand for robots in various application fields has been increasing in recent years. Robots are becoming active in a greater variety of environments, so there is a growing need for higher robot mobility that ensures safe and smooth movement. That need has drawn attention to the omnidirectional vehicle (ODV). ODV's can move forward and backward and turn to the left and right, but they can also move directly sideways and diagonally, and rotate in a fixed position. This high mobility enables an ODV to change direction in a small space, to parallel park easily, and perform other adaptive movements that are suitable to the vehicle environment. Omni-directional mobility has previously required special mechanisms that have been associated with problems of durability and stability. To address those problems, we have developed a nonholonomic ODV that uses two-wheel modules equipped with ordinary tires. However, the stability and smooth motion of the vehicle is usually affected by the load from the road surface, so a control system that distributes that load appropriately over all the motors is needed. Another problem is that there may be a loss of total driving force and vehicle speed under conditions in which the tires spin freely without traction. To maintain total driving force, a torque compensation system for each of the wheels is important. Here, we propose a method for load distribution control and a method for skid compensation control and present test results that show the effectiveness of the proposed methods.
\end{abstract}

Key words : Omni-directional vehicle, Multiple mobile robots, Distributed coordination, Environmental load distribution, Skid detection

\section{Introduction}

The demand for robots in society has been increasing in recent years. Because of the diversification of the environments in which robots are being used according to the needs of people, there has been much research on high robot mobility that enables robots to move safely and smoothly, even in unknown environments. Omnidirectional vehicles (ODV) have been attracting attention for that purpose. An ODV can move in any direction and turn while maintaining the same attitude, so application in many fields is expected. For example, an electrical wheelchair that has omnidirectional mobility has been proposed by Kitagawa et al (2004), and Okumura et al (2007) have proposed a passenger vehicle that is capable of omnidirectional motion as a concept for the development of next-generation vehicles. Other examples include an autonomous mobile robot (Tang et al, 1999) an a planetary exploration robot (Otsuki et al., 2011).

Omnidirectional motion requires special mechanisms, and most previous research has resulted in proposals and development of special wheels and mechanisms that are suited to the use environment (Tadakuma, 2011). However, ODVs that use special wheels have posed problems of durability and drivability because of structural complexity. The active caster mechanism developed by Wada (2007) uses pneumatic tires, so drivability is better, but it is necessary to successively update the vehicle kinematics according to the operating situation. Also, different motors are needed for running and steering, so the efficiency of motor use is low.

Considering problems such as those described above, our research group has developed an ODV that uses modular 
driving units. That ODV has a structure that offers higher drivability than previous special mechanisms and is not affected by road surface conditions. It also has higher efficiency of motor usage than the active castor mechanism that uses pneumatic tires. However, with conventional running control, the effects of the environmental load on the ODV and skid of the tires are not taken into account, so it may be difficult to achieve stable operation on uneven ground outdoors. Ishigami et al.,(2011) developed a vehicle assuming use on uneven outdoors terrain, but they used a mechanical approach to ensure stability by adding a suspension mechanism, etc. rather than taking a control approach. The generally-known control approaches to stability include use of a disturbance observer (DOB) or a reaction torque observer (RTOB). Usuki et al., (2014) have proposed a load distribution method that uses cooperative control and an RTOB for ODVs. That method, however, cannot distribute load over the entire vehicle according to the direction of motion and does not take ODV kinematics during turns into account. For tire slippage, traction control (Yoshimura et al, 2011) and other methods have been effective, but conventional traction control mostly controls tire slippage by actively decreasing torque, and so does not effectively solve the problem of reduction in overall vehicle driving force (Maeda et al., 2012).

To address the problems described above, we propose a method for distributing motor load under excessive load on the ODV without impeding driving control and a method for maintaining constant total driving force when wheel skid occurs.

In section 2, we present a control method for omnidirectional motion. In section 3, we describe the proposed methods, and in section 4 we describe tests in which actual machines were used. Section 5 concludes this paper.

\section{Control for Omnidirectional Motion}

\subsection{Vehicle model}

The proposed ODV has four wheel modules. A wheel module has two ordinary pneumatic tires that are driven independently. A translational motion is obtained by driving the two wheels (left and right) equally in the same direction and turning is obtained by driving the wheels at different speeds. The ODV has a total of eight wheel mechanisms for the four two-wheel modules, with redundant degrees of freedom in planar motion. Thus, movement in any direction on a plane can be achieved by cooperative control of the attitude angle and speed of each wheel module (Fig. 1).

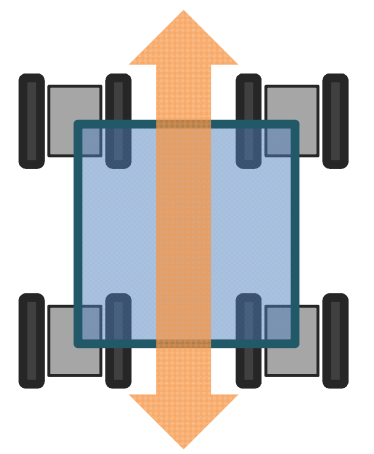

(a) Forward and backward motion (b) Left and right motion

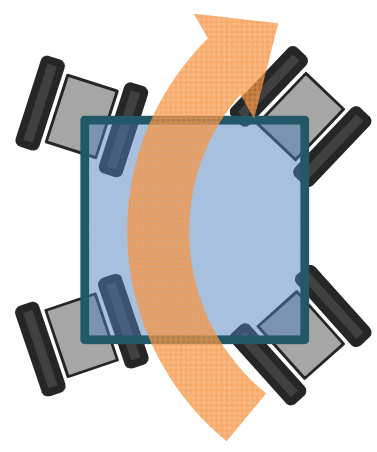

(c) Turning

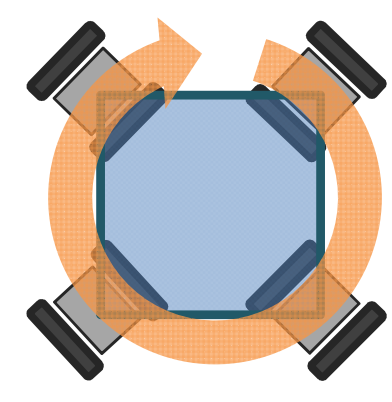

(d) Rotation in place

Fig. 1. Movement models. (a) and (b) are translations, and (c) and (d) are turns.

\subsection{Command values}

Because ODV operation in planar motion requires input for three degrees of freedom, we assume a controller that has a three-axis joystick. For intuitive operation by the operator, we set the $\mathrm{x}$-axis input as the speed in the $\mathrm{x}$ axis direction of the vehicle coordinate system, the $y$-axis input as the speed in the $y$-axis direction, and the $z$-axis input as the amount of vehicle turning. The ODV command values are speed $v^{\text {cmd }}$, translation direction $\phi^{\text {cmd }}$, and turn radius $r^{c m d}$. The values are calculated using the following equations.

$$
v^{c m d}=C_{1} \sqrt{X_{i n}^{2}+Y_{i n}^{2}}
$$




$$
\begin{aligned}
& \phi^{c m d}=\tan ^{-1}\left(\frac{X_{i n}}{Y_{i n}}\right) \quad\left(-\pi \leq \phi^{c m d}<\pi\right) \\
& \kappa=C_{2} Z_{i n} \\
& r^{c m d}=\left|\frac{1}{\kappa}\right|
\end{aligned}
$$

We denote the input values of the three-axis joystick as $X_{i n}, Y_{i n}$, and $Z_{i n}$, and the gain values as $C_{1}$ and $C_{2}$. Because the turn radius is infinitely large for translation motion, we calculate the inverse curvature $\kappa$ in Eq. (3) and then convert to the turn radius for the command value $r^{\text {cmd }}$.

\subsection{Wheel module reference values}

For translation motion, which is to say the case in which there is no z-axis input from the joystick, the attitude angle $\phi_{n}^{\text {ref }}$ and speed $v_{n}^{\text {ref }}$ of each wheel module are the same as the command values.

$$
\begin{aligned}
& \phi_{n}^{r e f}=\phi^{c m d} \\
& v_{n}^{r e f}=v^{c m d}
\end{aligned}
$$

Next, consider the case in which the ODV is turning. For smooth turning, the wheels must be oriented so that straight lines extended from each of the vehicle axles should cross at one point. This turning method is known as the Ackermann geometry (Choi et al., 2008) and the crossing point is called the center of turning circle. When Ackermann geometry is used, the turning radius $r_{n}$ and attitude angle $\phi_{n}^{\text {ref }}$ for each wheel module are calculated as follows.

$$
\begin{aligned}
& r_{n}=\sqrt{\left(r^{c m d} \cos \phi^{c m d} \pm x_{n}\right)^{2}+\left(r^{c m d} \sin \phi^{c m d} \pm y_{n}\right)^{2}} \\
& \phi_{n}^{r e f}=\tan ^{-1}\left(\frac{r^{c m d} \sin \phi^{c m d} \pm y_{n}}{r^{c m d} \cos \phi^{c m d} \pm x_{n}}\right)
\end{aligned}
$$

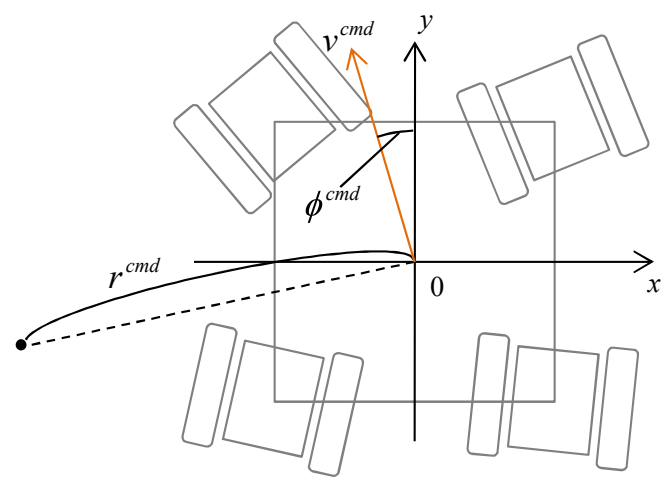

(a) ODV command values

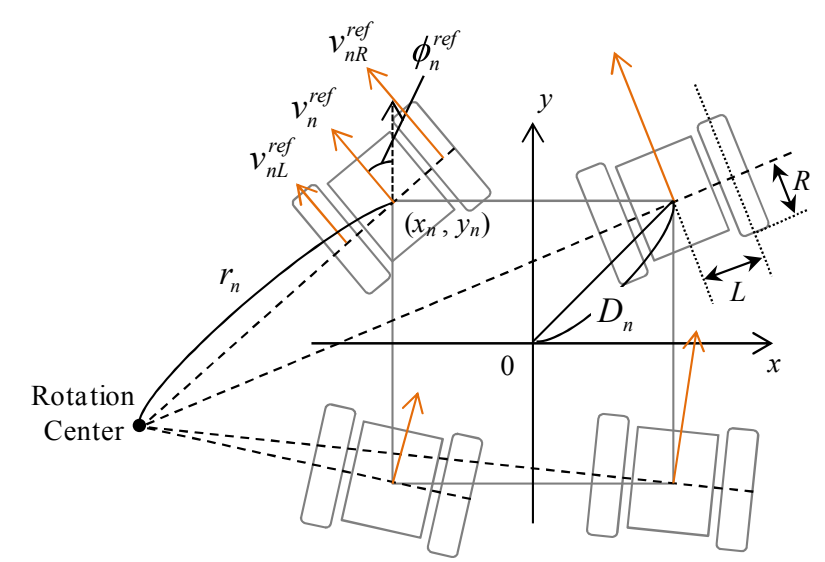

(b) Ackermann geometry

Fig. 2. Vehicle and wheel module models 
In a relative coordinate system whose origin is the center of the vehicle, let $x_{n}$ be the $\mathrm{x}$ direction distance and let $y_{n}$ be the $y$ direction distance from the vehicle center to the turning axis of a wheel module. Because the geometrical relationship of the inner and outer wheels changes in a turn to the left and a turn to the right, the sign in the equation changes according to the $\mathrm{z}$-axis input. The speed of each wheel module is calculated with Eq. (9) according to the turn radius. The speeds of the left and right wheels, $v_{n L}^{r e f}$ and $v_{n R}^{r e f}$, are calculated with Eq. (10), with $L$ denoting half of distance between the wheels. For rotating in place, left and right wheel speeds are obtained by making the turn radius the distance $D_{n}$ from the vehicle center to the turning axis of each wheel module.

$$
\begin{aligned}
& v_{n}^{r e f}=\frac{r_{n}}{r^{c m d}} v^{c m d} \\
& {\left[\begin{array}{c}
v_{n R}^{r e f} \\
v_{n L}^{r e f}
\end{array}\right]=\left[\begin{array}{c}
\frac{r_{n} \pm L}{r_{n}} \\
\frac{r_{n} \mp L}{r_{n}}
\end{array}\right] v_{n}^{r e f}=\left[\begin{array}{c}
\frac{r_{n} \pm L}{r^{c m d}} \\
\frac{r_{n} \mp L}{r^{c m d}}
\end{array}\right] v^{c m d}}
\end{aligned}
$$

\section{Proposed Methods}

\subsection{Load distribution control}

Cooperative control for each wheel module alone does not take the effect of load on the vehicle into account, so tracking performance on uneven terrain is poor. For that reason, we define the load deviation for each wheel module and propose a control that distributes excessive load on a wheel module evenly over all wheel modules.

\subsubsection{Definition of load deviation}

We define two indices of load deviation for each wheel module. First, we consider the ratio of the maximum continuous torque of the motor to the external torque estimated by the RTOB and define a value that indicates the balance of load on the wheel modules as Eq. (11). We will refer to the value defined by Eq. (11) as the $L B_{n}$ value.

$$
L B_{n}=\frac{\left|\hat{\tau}_{m, n}^{\text {ext }}\right|}{\tau_{m, n}^{\max }}-\frac{\sum_{k=1}^{N}\left|\hat{\tau}_{m, k}^{\text {ext }}\right|}{n \tau_{m, n}^{\max }}
$$

The term $\tau_{m, n}^{\max }$ is the maximum continuous torque of the motor and each module has the same value. $\hat{\tau}_{m, n}^{\text {ext }}$ is the external torque on the wheel module estimated by the RTOB, $\mathrm{n}$ is a number that identifies the wheel module, and $N$ is total number of modules. Equation (11) is an extension of the method proposed by Tsuji et al.,(2004); its value is positive when the load is greater than the load on the other wheel modules and negative when the load is less than the load on other modules. The value of $L B_{n}$ contributes greatly to the load distribution torque, which is described later in this paper.

The second index of load deviation is an evaluation function that expresses the overall load balance of the ODV as defined by Eq. (12).

$$
E L B=\sum_{k=1}^{N}\left(\frac{\left|\hat{\tau}_{m, k}^{\text {ext }}\right|}{\tau_{m, k}^{\max }}-\frac{\sum_{l=1}^{N}\left|\hat{\tau}_{m, l}^{\text {ext }}\right|}{n \tau_{m, k}^{\max }}\right)^{2}=\sum_{k=1}^{N} L B_{k}^{2}
$$

Equation (12) is an extension of the method proposed by Sasaki et al.,(1996) to this ODV. The value of ELB is zero 
when the load is completely distributed evenly over all of the wheel modules. That is to say, the objective of the work reported here is keeping the value of $E L B$ close to zero.

\subsubsection{Load distribution torque}

The input torque to a wheel module that is experiencing excessive load must be increased so as to offset the load. However, simply increasing the input torque will create a partial vehicle acceleration that can have a bad effect on the overall movement of the ODV. Accordingly, appropriate distribution of the environmental load requires that the torque be redistributed by decreasing the input torque to motors that have less load and increasing the torque to the motors that are under heavier load by that same amount. To satisfy those requirements, a torque that corresponds to the value of $L B_{n}$ is applied at each wheel module to eliminate the load deviation. The load distribution torque $\tau_{n}^{\text {dist }}$ that is generated at each wheel module is defined by Eq. (13), in which $C^{\text {dist }}$ is the gain. By adding the load distribution torque $\tau_{n}^{\text {dist }}$ to the input of each motor, the load deviation for the ODV as a whole can be eliminated and the ELB value can be lowered. A block diagram of this load distribution control is shown in Fig. 3.

$$
\tau_{n}^{\text {dist }}=C^{\text {dist }}\left(\left|\hat{\tau}_{m, n}^{\text {ext }}\right|-\frac{\sum_{k=1}^{N}\left|\hat{\tau}_{m, k}^{\text {ext }}\right|}{N}\right)
$$

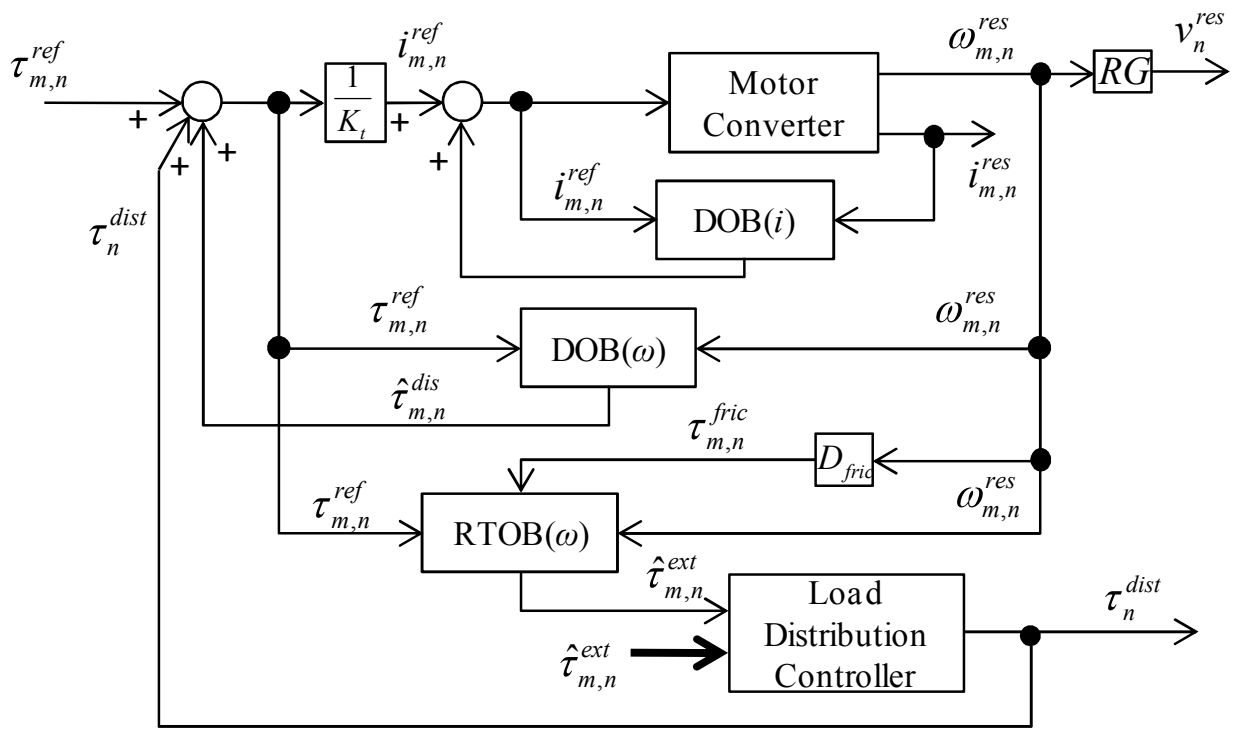

Fig. 3. Block diagram of the load distribution control system; RTOB $(\omega)$ is a reactive torque observer that uses angular velocity and $\tau_{n}^{\text {dist }}$ is the load distribution torque that is calculated when an excessive load is applied to a wheel module.

\subsection{Skid compensation control}

This ODV is designed for eight driving wheels and has eight points of contact with the ground surface. During operation, however, if there is wheel skid, the total ODV driving force decreases and the operator unintentionally decreases the speed. We therefore propose here a method for detecting wheel skid and a method for maintaining total driving force when skid occurs.

\subsubsection{Skid detection}

Kadowaki et al., (2005) use the DOB to estimate a tangential force coefficient $\hat{\mu}$ and use that value to identify wheel skid. It is known that $\hat{\mu}$ decreases suddenly when a wheel skid during operation (Ohishi, 1999). For a more robust indicator, we add an RTOB to the DOB and propose a skid detection method that uses a disturbance torque $\hat{\tau}_{m, n k}^{\text {dis }}$ and an external torque $\hat{\tau}_{m, n k}^{\text {ext }}$ which are linear with respect to $\hat{\mu}$.

In the detection method that uses the DOB (Fig. 4), a skid region is set for the estimated disturbance torque $\hat{\tau}_{m, n k}^{\text {dis }}$ and skid is judged to be occurring when the value remains within that region for a certain time. The skid function 
$\varepsilon_{n k}^{D O B}$ defined by Eq. (14) takes the value of 1 when skid is judged to occur or the value of 0 when the wheel is in contact with the ground.

$$
\varepsilon_{n k}^{D O B}=\left\{\begin{array}{cc}
1 & \left(\tau_{\text {Low }}^{\text {dis }} \leq \hat{\tau}_{m, n k}^{\text {dis }}<\tau_{H i g h}^{d i s}\right) \\
0 & \text { otherwise }
\end{array}\right.
$$

Next, we consider a method that introduces an RTOB (Fig. 5). The RTOB provides a purer estimate of the environmental reactive force by subtracting the viscous friction term from the external disturbance estimated by the DOB. A region for detecting the skid state is also set for the external torque $\hat{\tau}_{m, n k}^{\text {ext }}$ estimated by the RTOB. The skid function used by the RTOB $\varepsilon_{n k}^{R T O B}$ is defined by Eq. (15), and the final skid function $\varepsilon_{n}$ is defined by Eq. (16).

$$
\begin{gathered}
\varepsilon_{n k}^{\text {RTOB }}=\left\{\begin{array}{cc}
1 & \left(\tau_{\text {Low }}^{\text {ext }} \leq \hat{\tau}_{m, n k}^{\text {ext }}<\tau_{\text {High }}^{\text {ext }}\right) \\
0 & \text { otherwise }
\end{array}\right. \\
\varepsilon_{n}=\left\{\begin{array}{cc}
1 & \left(\varepsilon_{n k}^{D O B}=\varepsilon_{n k}^{\text {DTOB }}=0\right) \\
0 & \text { otherwise }
\end{array}\right.
\end{gathered}
$$

We know from experiments that the skid functions in the above equations transition more easily to 0 than to 1 . Therefore, when the skid functions of Eq. (14) and Eq. (15) have different values, we can expect a reduction in detection error by using the function whose value is 1 .

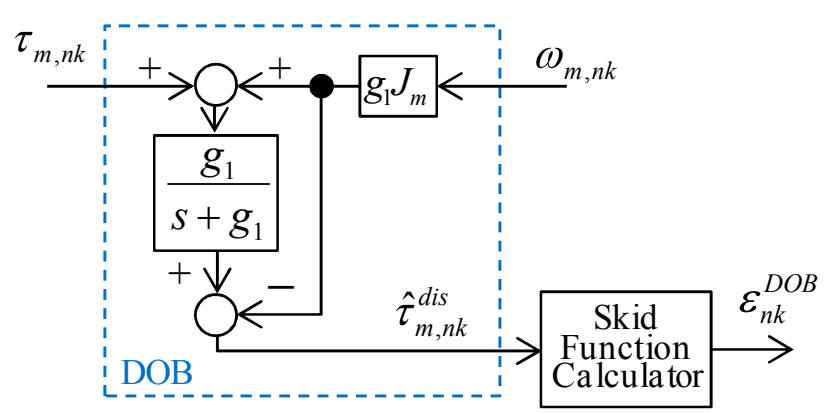

Fig. 4. Skid detection using DOB

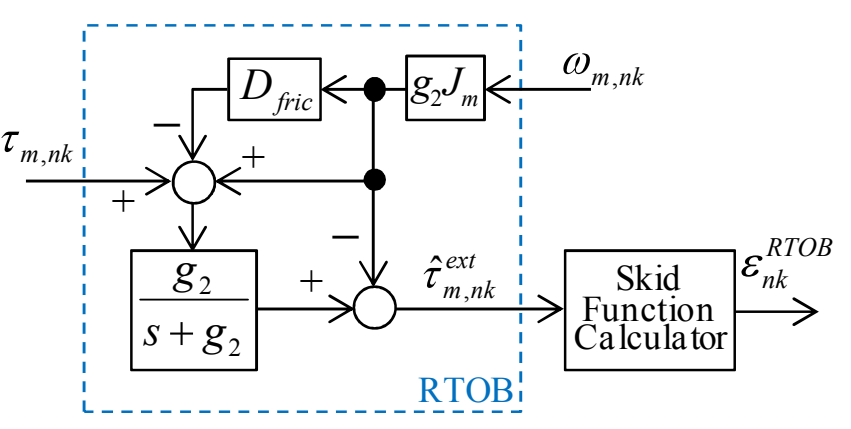

Fig. 5. Skid detection using RTOB

\subsubsection{Calculation of compensation torque and speed for wheel skid}

Maintaining a constant total ODV driving torque requires compensation of insufficient driving torque to wheel modules that are touching the ground when skid of any of the wheels is detected. We therefore define the compensation torque for wheels that are in contact with the ground $\tau_{m, n}^{c o m}$ with the following equation. We will refer to this value as the skid compensation torque.

$$
\begin{aligned}
\tau_{m, n}^{*} & =\varepsilon_{n}\left(\tau_{m, n L}^{r e s}+\tau_{m, n R}^{r e s}\right) \\
\tau_{m, n}^{c o m} & =\frac{\sum_{k=1}^{N} \tau_{m, k}^{*}}{N_{p}}
\end{aligned}
$$

In the above equations, when an n-th module skids, $\varepsilon_{n}$ becomes 1 , and therefore $\tau_{m, n}^{*}$ becomes the driving torque to decrease. And $N_{p}$ is the number of wheel modules that are touching the ground. However, this ODV has a speed control, so a fast torque response cannot be achieved if we simply add the skid compensation torque $\tau_{m, n}^{c o m}$ to the 
motor input. For that reason, we must convert the skid compensation torque $\tau_{m, n}^{\text {com }}$ to the skid compensation velocity $v_{n}^{\text {com }}$, and apply it from outside the speed feedback loop. The following equation is used to convert from torque to velocity.

$$
v_{n}^{t m p}=c_{n}^{c o m} G \frac{R}{J_{n}} \tau_{n}^{c o m} \Delta t
$$

In the above equation, $G$ is the deceleration ratio, $R$ is the wheel radius, $J_{n}$ is the inertial moment of the wheel, and $\Delta t$ is the addition time. Mechanically, the conversion of torque to speed involves integration, but for real-time response we can maintain consistency with integration calculations by adjusting the gain $c_{n}^{\text {com }}$. However, the velocity calculated with Eq. (19) does not take Ackermann geometry into account, so cooperative movement of the wheel modules during a turn is lost. Using the ODV turn command $r^{\text {cmd }}$ and the turn radius $r_{n}^{r e f}$ of each wheel module, the appropriate skid compensation velocity to be applied to each wheel module, $v_{n}^{\text {com }}$ is given by Eq. (20). A block diagram for the skid compensation control is shown in Fig. 6.

$$
v_{n}^{c o m}=v_{n}^{t m p} \frac{r_{n}^{r e f}}{r^{c m d}}
$$

The same calculation about all modules are performed.

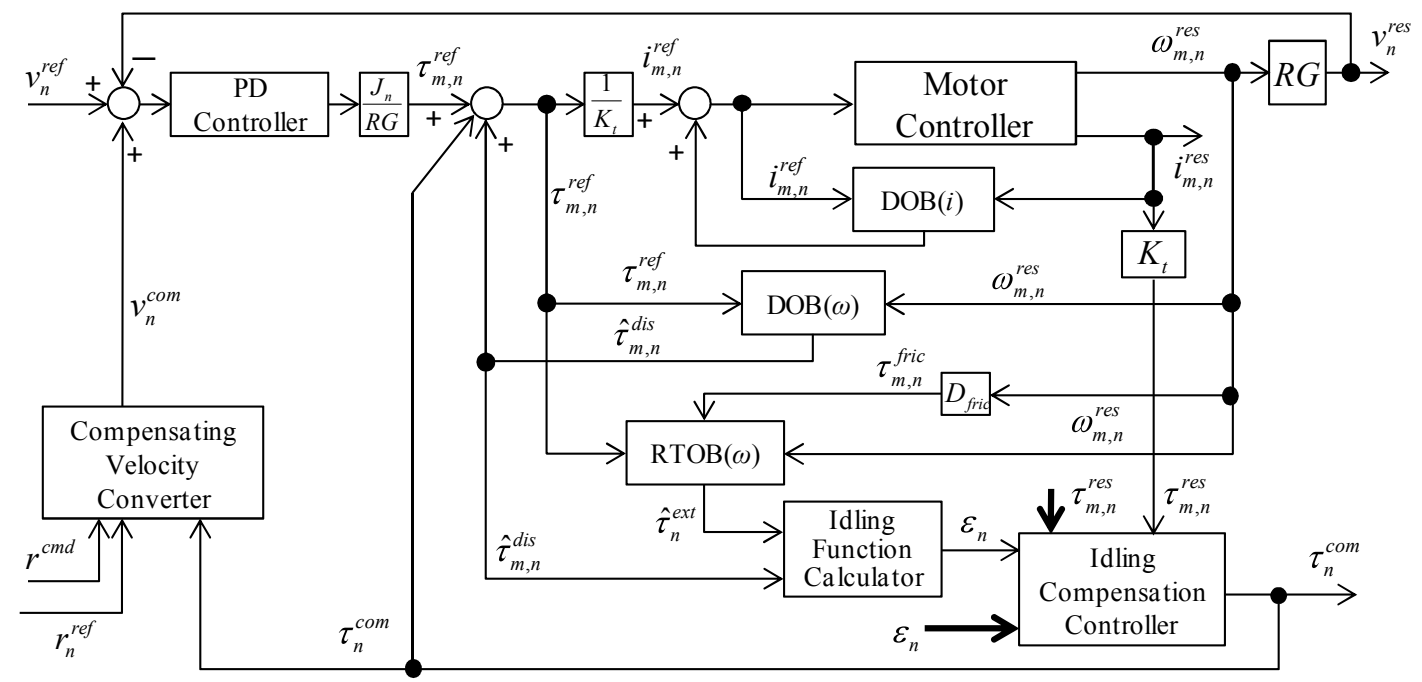

Fig. 6. Block diagram for the skid detection and compensation system; $\varepsilon_{n}$ is the skid function and $\tau_{n}^{\text {com }}$ is the skid compensation torque that is calculated for a wheel module when any tire is skid.

\subsubsection{Single-wheel attitude angle control and total driving torque}

Suitable operation is difficult when one of the two wheels of a wheel module is skid, because it produces a rotation vector around the turning axis, and cooperative wheel attitudes cannot be maintained. We therefore introduce a control that corrects the attitude angle of the wheel that is touching the ground in a wheel module whose left or right wheel is skid so that the attitude is always in the direction of ODV motion. This control incorporates a PID control that takes the skid function as a variable gain and is called a one-wheel attitude angle control. A block diagram for this control is shown in Fig. 7. However, the driving force cannot be generated by simply correcting the attitude angle of a wheel module that has one skid wheel. The total driving force on the ODV is defined by Eq. (21). 


$$
\tau^{\text {total }}=\sum_{k=1}^{n}\left(1-\varepsilon_{k}\right)\left(\tau_{k L}^{r e s}+\tau_{k R}^{r e s}\right)
$$

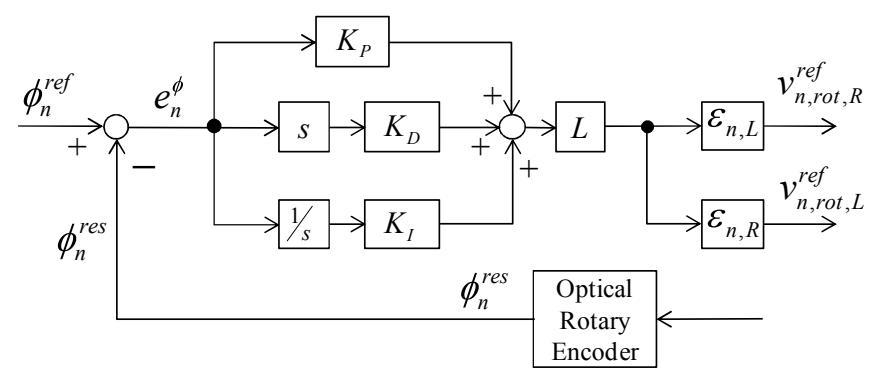

Fig. 7. Block diagram for one-wheel attitude angle control

\section{Experiments}

\subsection{ODV structure}

The ODV developed by our research group comprises a control device and four wheel modules. The control device has a three-axis joystick, a CPU board (SH7216, $200 \mathrm{MHz}$ ), and an OLED that displays the data that is processed internally. Each wheel module is also equipped with a same CPU board, and so is itself a robot that can move autonomously. The attitude angle and speed of each wheel module are controlled appropriately by command values issued by the control device. The box unit of the wheel module contains a battery and motor. The CPU board, power supply board and motor driver are mounted on the front and back walls of the unit. The wheel modules are connected to the chassis with free joints, so each wheel module can rotate around a vertical axis without constraint. An optical rotary encoder used to measure the attitude angle is attached here. The appearance of the ODV is shown in Fig. 8 and the specifications are listed in Table 1.

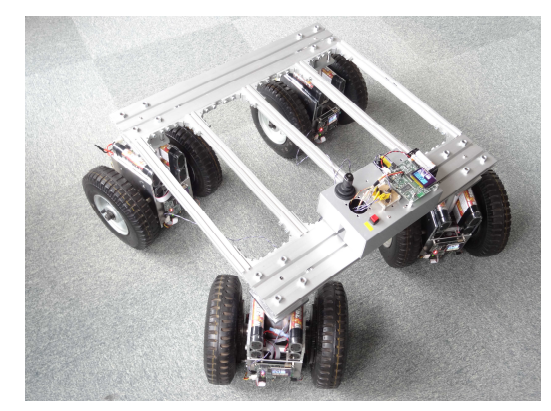

Table 1. Specifications of the proposed ODV

\begin{tabular}{|c|c|}
\hline Overall length & $610 \mathrm{~mm}$ \\
\hline Overall width & $640 \mathrm{~mm}$ \\
\hline Overallheight & $325 \mathrm{~mm}$ \\
\hline Wheelbase & $500 \mathrm{~mm}$ \\
\hline Tread & $500 \mathrm{~mm}$ \\
\hline Diameter of tire & $220 \mathrm{~mm}$ \\
\hline Width of rim & $60 \mathrm{~mm}$ \\
\hline Gross weight & $44.5 \mathrm{~kg}$ \\
\hline Weight of wheelmodule & $9.8 \mathrm{~kg}$ \\
\hline
\end{tabular}

Fig. 8. Appearance of the proposed ODV

\subsection{Experimental results of Omnidirectional motion tests}

We performed running experiments to test the omnidirectional movement performance of the developed ODV. The

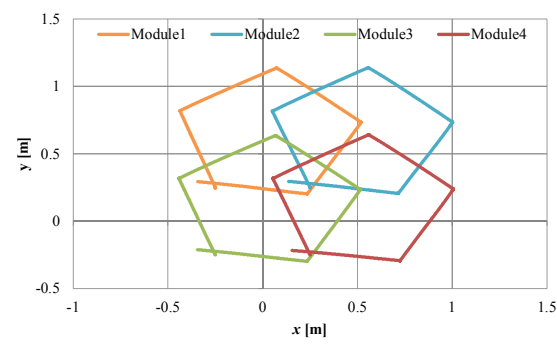

(a) Translation (regular pentagon)

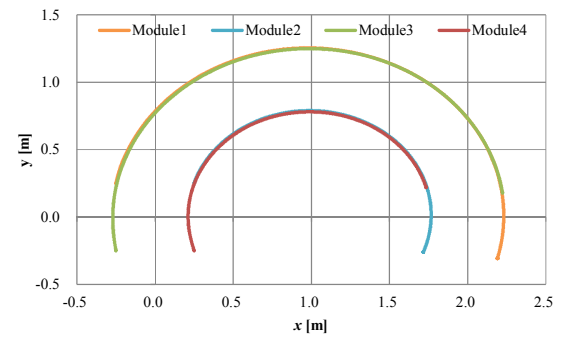

(b) Turning

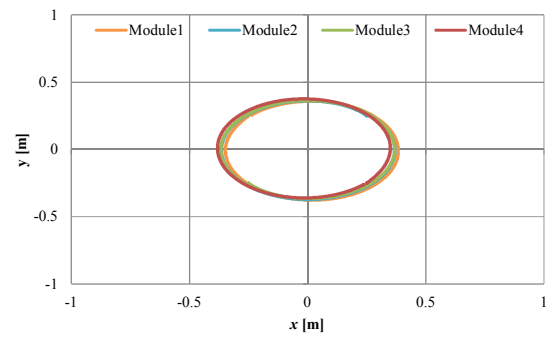

(c) Rotating in place

Fig. 9. Results of running tests using actual device 
trajectories of the wheel modules for translation motion, turning, and rotation in place are shown in Fig. 9. The sampling time used in these tests was $10 \mathrm{~ms}$. These results confirm the omnidirectional motion performance of the developed ODV.

\subsection{Load distribution tests}

To test the effectiveness of the load distribution control, we performed step-passing tests and slope-climbing tests. The test conditions were to send speed command values of $0.1 \mathrm{~m} / \mathrm{s}$ to the ODV from $5 \mathrm{~s}$ to $25 \mathrm{~s}$ with a sampling time of $10 \mathrm{~ms}$. The values of these limits in eq.(14) and eq.(15) are selected by experiments beforehand.

\subsubsection{Step-passing tests}

To test the effect of an instantaneous excessive load on the ODV, we performed three step-passing tests (Fig. 10). The initial arrangement of the ODV and the step was such that at $10 \mathrm{~s}$ after the OVD began to move forward, the front wheels began to climb the step and at $15 \mathrm{~s}$ the rear wheels began the climb. At $17 \mathrm{~s}$, the front wheels began to descend the step, followed by the rear wheels at $22 \mathrm{~s}$. The change in ELB value for when the ODV passes over the 17.5-mm step is shown in Fig. 11. With the previous method, the ELB value rises sharply when the wheels ascend and descend the step; with the proposed method, the sharp rises in $E L B$ value are suppressed and the overall ELB values are also lower. This result confirms the overall load distribution effect of the proposed method.

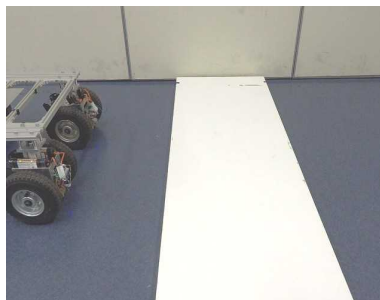

(a) $0 \mathrm{~s}$

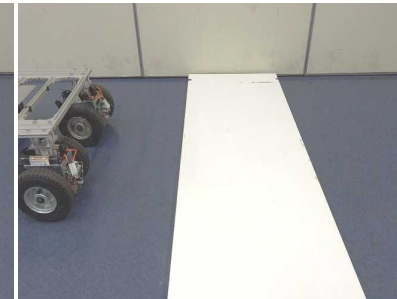

(b) $5 \mathrm{~s}$

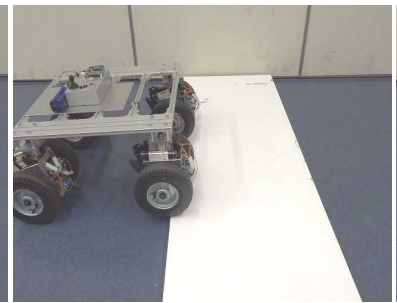

(c) $10 \mathrm{~s}$

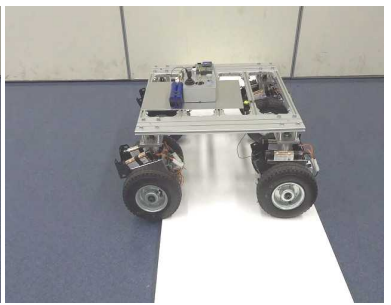

(d) $15 \mathrm{~s}$

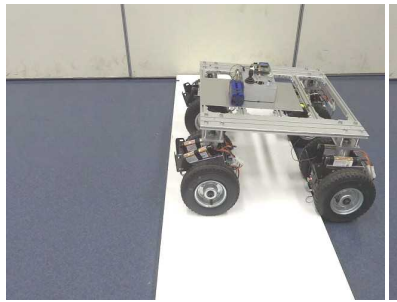

(e) $17 \mathrm{~s}$

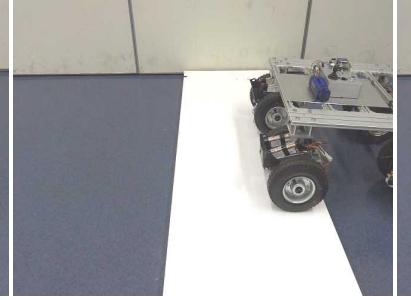

(f) $20 \mathrm{~s}$

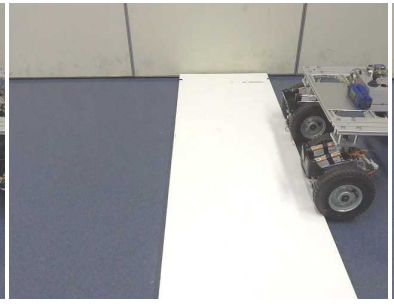

(g) $22 \mathrm{~s}$

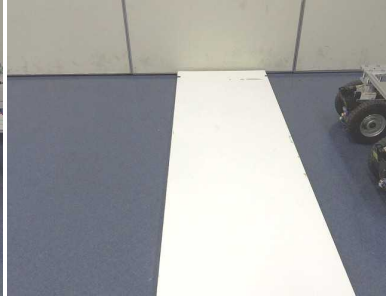

(h) $25 \mathrm{~s}$

Fig. 10. Step-passing tests
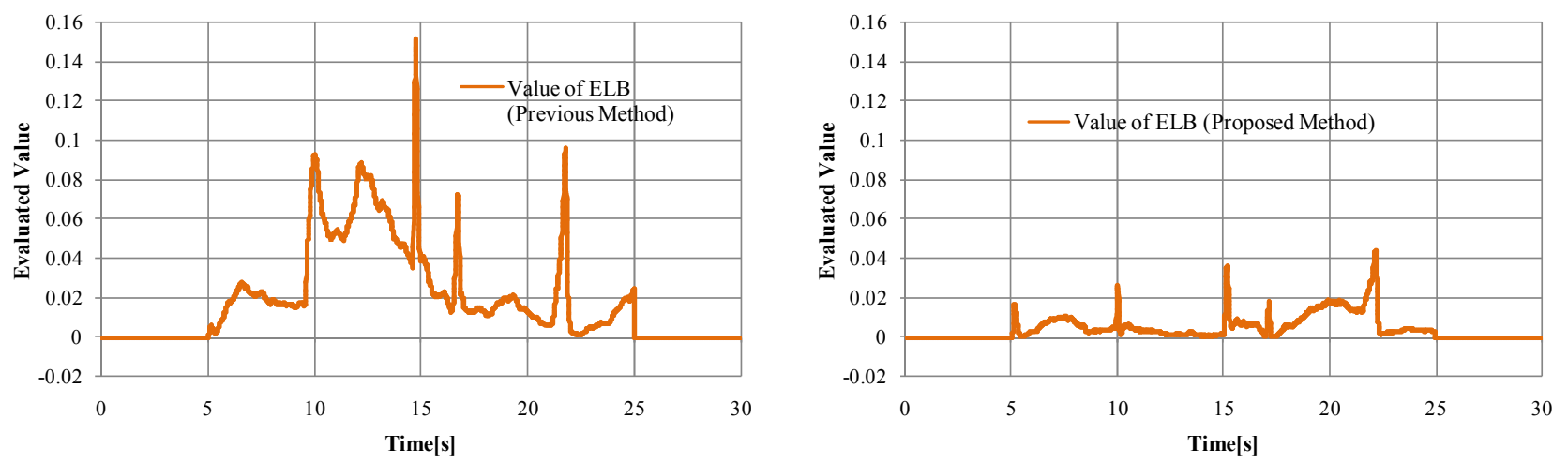

Fig. 11. Results of step-climbing test A; the lower maximum ELB of the proposed method indicates an improvement over the previous method. 
The maximum ELB values for each test are compared in Fig. 12. The step heights were $17.5 \mathrm{~mm}$ in Experiment A, $35.0 \mathrm{~mm}$ in Experiment B, and $52.5 \mathrm{~mm}$ in Experiment $\mathrm{C}$. The ratios of the results for the proposed method to the results for the previous method are $29.1 \%$ for Experiment A, 14.5\% for Experiment B, and 5.20\% for Experiment $\mathrm{C}$, showing that the maximum $E L B$ was lower for the proposed method in all tests. These results confirm the effectiveness of the load distribution control when an instantaneous excessive load is applied.

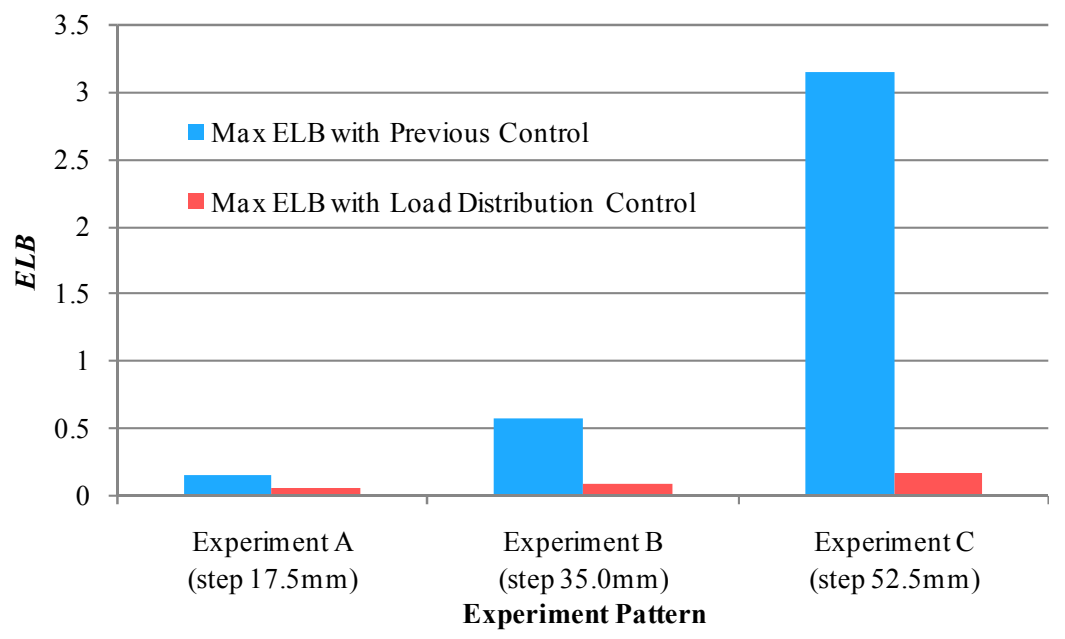

Fig. 12. Results for all step-passing tests, showing the maximum ELB values for the previous and proposed methods

\subsubsection{Slope climbing tests}

We performed slope climbing tests with a 5-degree inclined plane to verify ODV operation under a continuous load (Fig. 13). The change in ELB values for operation on an outdoors slope is shown in Fig. 14. In the same way as for the step-passing test, the proposed method resulted is lower $E L B$.

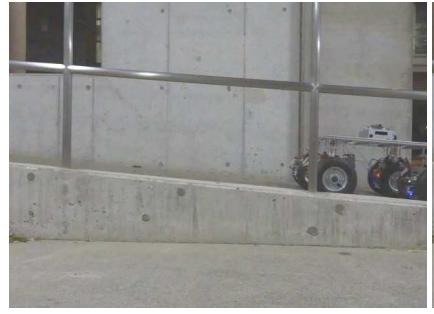

(a) $0 \mathrm{~s}$

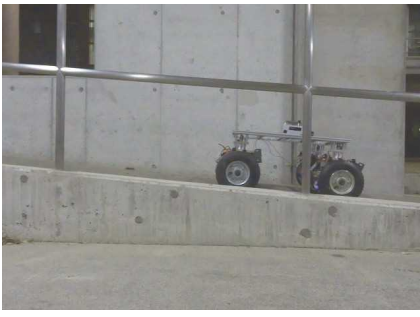

(b) $10 \mathrm{~s}$

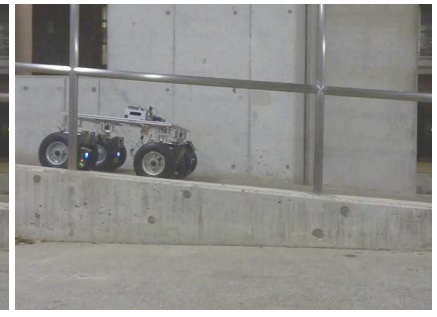

(c) $20 \mathrm{~s}$

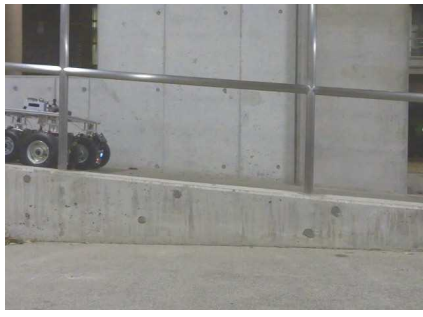

(d) $30 \mathrm{~s}$

Fig. 13. Slope climbing tests

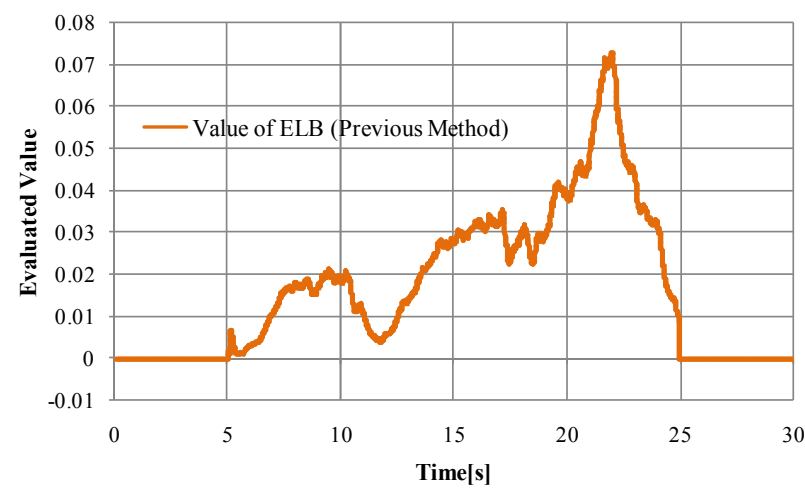

(a) Previous method

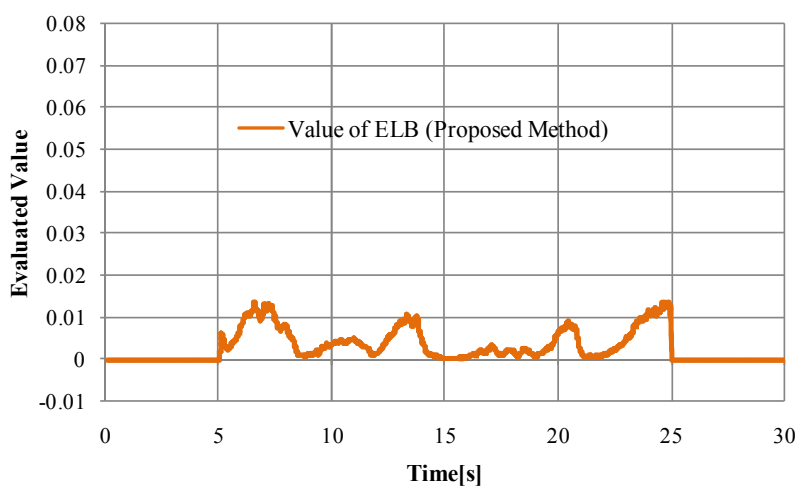

(b) Proposed method

Fig. 14. Results for slope-climbing test D; the lower maximum ELB of the proposed method indicate an improvement over the previous method. 
Next, we consider the average ELB values for each of the tests (Fig. 15). Experiment D and E are respectively for outdoor and indoor slopes. Experiment $\mathrm{F}$ is for motion at a $45^{\circ}$ angle relative to an indoor slope. The ratios of the results for the proposed method to the results for the previous method are $18.0 \%$ for Experiment $\mathrm{D}, 38.0 \%$ for Experiment E, and $34.1 \%$ for Experiment F. The average ELB was lower for the proposed method in all tests. These results confirm the effectiveness of the load distribution control under continuous load.

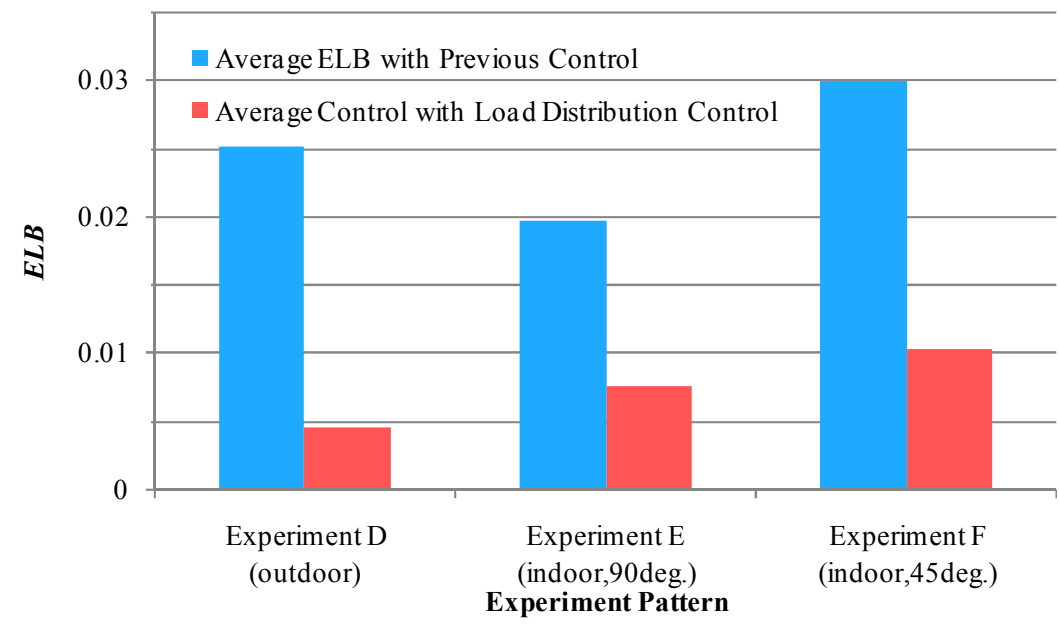

Fig. 15. Results for all slope climbing tests; average $E L B$ values are shown for the previous and proposed methods.

\subsection{Skid compensation tests}

To test the effectiveness of the skid compensation control, we performed experiments in which there was wheel skid during operation of the ODV. We placed mats that have short bristles on the floor to induce wheel skid and performed four types of tests that involved translation motion and turning. The sampling time was $10 \mathrm{~ms}$ and speed commands were sent to the ODV at intervals of from $2 \mathrm{~s}$ to $8 \mathrm{~s}$.

The situation of the skid compensation test for translation motion is shown in Fig. 16. The total driving torque for the case of one wheel skid in translational motion is shown in Fig. 17. In this test, the total driving torque for operation under the same conditions with all of the tires touching the ground was taken to be the theoretical value. With the previous method, the total driving torque was below the theoretical value while skid of wheel module 1 was detected, but the value remained about the same as the theoretical value when the proposed method was applied.

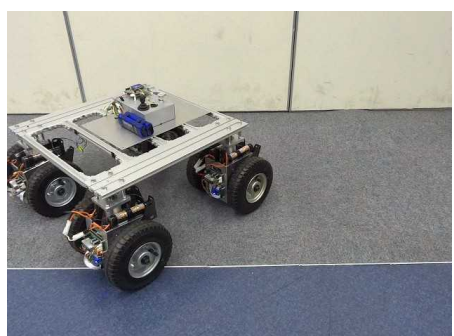

(a) $0 \mathrm{~s}$

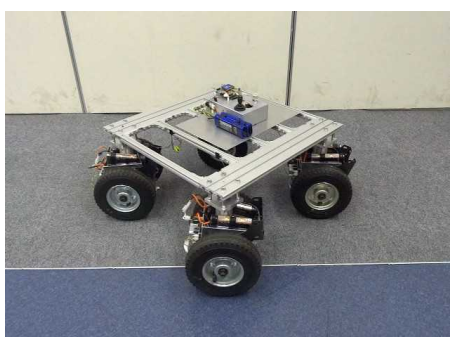

(d) $6 \mathrm{~s}$

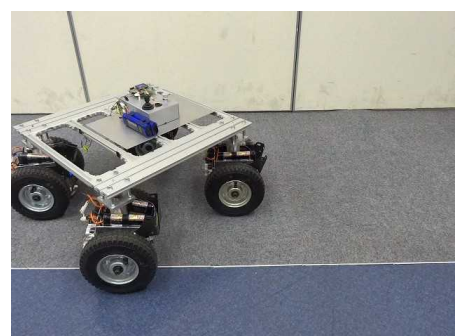

(b) $2 \mathrm{~s}$

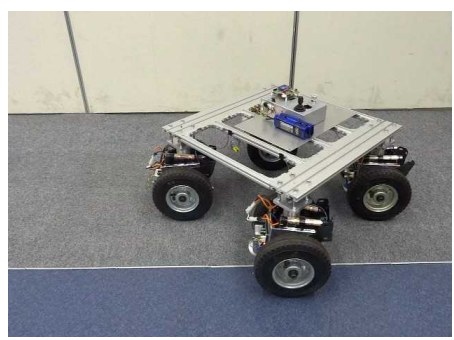

(e) $8 \mathrm{~s}$

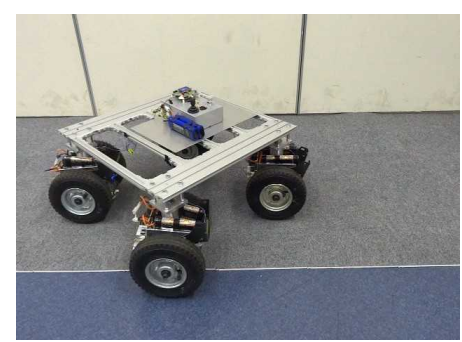

(c) $4 \mathrm{~s}$

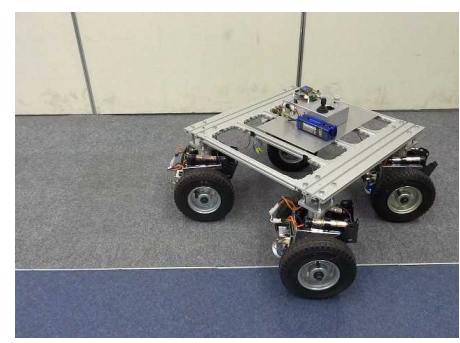

(f) $10 \mathrm{~s}$

Fig. 16. Free-spinning compensation experiments 


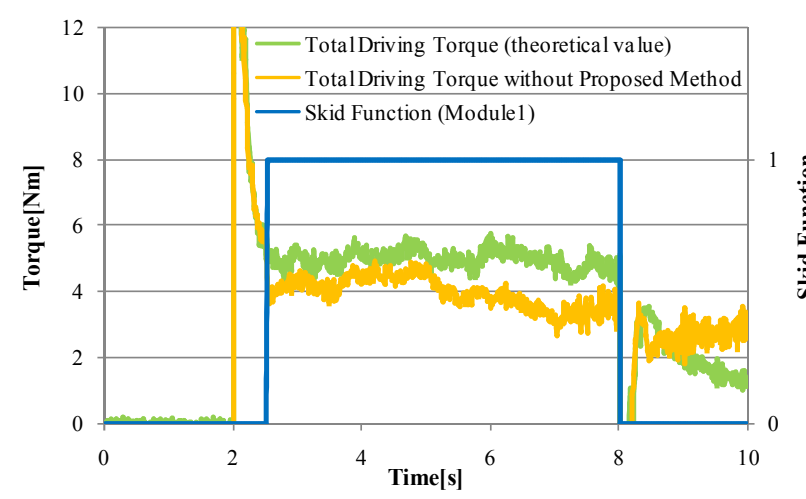

(a) Previous method

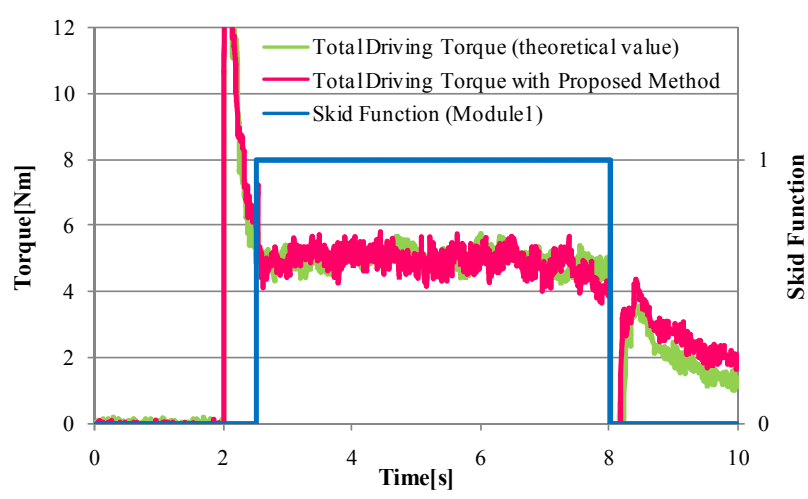

(b) Proposed method

Fig. 17. Results for wheel skid Experiment G; (a) shows the total drive torque without the proposed method (yellow), the theoretical torque (green), and the skid function (blue). (b) additionally shows the total driving torque for the proposed method (red). The total driving torque decreases when skid occurs with the previous method, but remains about the same with the proposed method.

Next, we consider the reduction rates for the total driving torque in each test (Fig. 18). Test G is for one wheel skid during translational motion, test $\mathrm{H}$ is for two wheels skid during translational motion, test $\mathrm{I}$ is for one wheel skid during turning motion, and test $\mathrm{J}$ is for two wheels skid during turning motion. The torque reduction values for the previous control are $20.3 \%$ for test $\mathrm{G}, 42.6 \%$ for test $\mathrm{H}, 40.1 \%$ for test $\mathrm{I}$, and $49.9 \%$ for test $\mathrm{J}$. For the proposed method, on the other hand, the values were $0.998 \%$ for test $\mathrm{G}, 17.4 \%, 4.12 \%$ for test I, and $25.7 \%$ for test $\mathrm{J}$. We can see that the proposed method resulted in lower torque reduction rates during wheel skid in all of the tests.

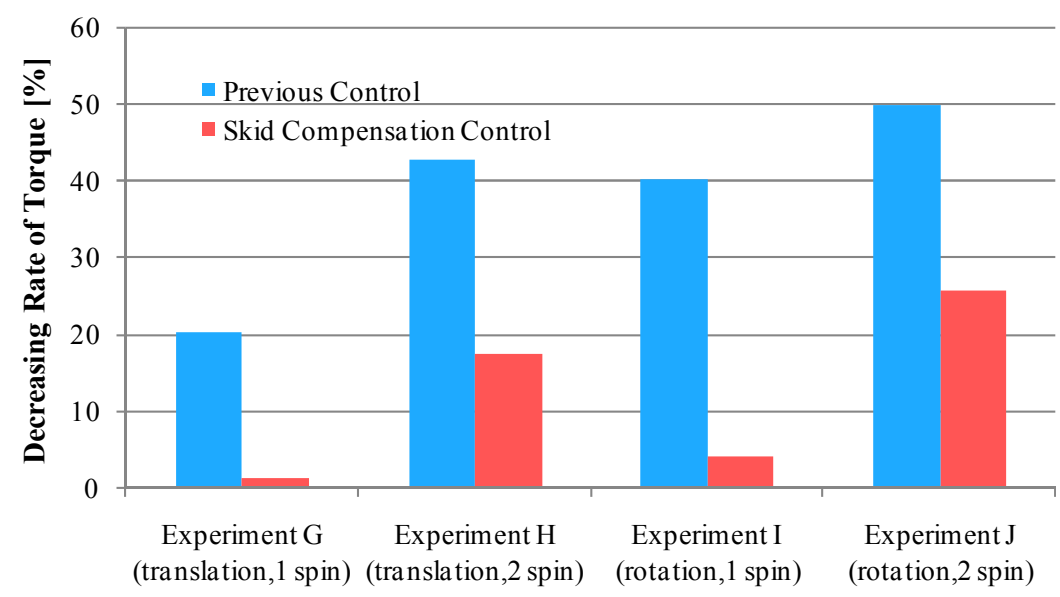

Experiment Pattern

Fig. 18 Comparison of torque decreasing rate by previous control and by skid compensation control

\section{Conclusion}

We have proposed control methods for free and efficient omnidirectional motion, focusing on an ODV that has independently driven wheel modules. The control system is a distributed system that consists of a control device that serves as the master controller and wheel modules that serve as subordinate controllers. The master controller calculates command values for the ODV as a whole and sends them to the subordinate controllers. When the command values are received, the subordinate controllers control the wheel module speed and attitude angle accordingly. The subordinate controllers implement special controls such as for sharp turn control, permitted movement region, and cooperative changing of attitude to achieve smooth and stable ODV movement.

In this paper, we have proposed two control systems for improving the stability of operation on uneven ground 
without improving the ODV mechanism itself. One is a method for evenly distributing load over all of the ODV wheel modules when environmental loading occurs during operation. The other is a method for maintaining the total driving torque of the ODV when wheel skid occurs.

The effectiveness of the methods we have proposed here was verified by developed ODV. Load distribution tests that included step-passing tests and slope climbing tests with large instantaneous loads and continuous loads verified that the proposed methods are effective under all test conditions. The skid compensation tests for single-wheel skid and two-wheel skid all confirmed that the proposed method resulted in less torque reduction for both translational movement and turning than did the previous method.

\section{References}

Choi, M. W., Park, J. S., Lee, B. S. and Lee, M. H., The performance of independent wheels steering vehicle(4WS) applied Ackerman geometry, 2008 International Conference on Control, Automation and Systems (ICCAS)(2008), pp.197-202.

Ishigami, G., Pineda, E., Overholt, J., Hudas, G., andIagnemma, K., Performance analysis and odometry improvement of an omnidirectional mobile robot for outdoor terrain, Intelligent Robots and Systems (IROS), 2011 IEEE/RSJ International Conference on, pp.4091-4096.

Kadowaki, S., Ohishi, K., Sano, T. and Yasukawa, S., Application of Anti-slip/skid Re-adhesion Control System Based on Disturbance Observer to a Skid Control Considering Cooperation Control of Air Bake and Electric Brake, IEEJ Transaction on Industry Applications, Vol.125, No.10 (2005), pp. 919-926 (in Japanese).

Kitagawa, H., Beppu, T., Ohno, Y., Miyoshi, T. and Terashima, K., Motion Control of Omni-directional Mobile Wheelchair Considering User's Comfort, Journal of Robotics Society of Japan, Vol.22, No.7 (2004), pp.933-939 (in Japanese).

Maeda, K., Fujimoto, H., Front and Rear Driving Force Distribution Method for Retaining Driving Force on Instantaneous Slippery Roads for Electric Vehicle with In-Wheel Motors, Transactions of the Japan Society of Mechanical Engineers, Series C, Vol.78, No.794 (2012-10), pp.3383-3392 (in Japanese).

Ohishi, K., Nakano, K., Miyashita, I. and Yashukawa, S., Anti-Slip Control of Electric Motor Coach Using Adhesion Force Coefficient Estimator Based on Disturbance Observer, IEEJ Transaction on Industry Applications, Vol.119-D, No.6 (1999.06.01), pp.802-808 (in Japanese).

Otsuki, M., Ishigami, G., Narita, Shinichiro and Wakabayashi, S., Proposal of control method for sinkage and vibration of planetary rover with flexible structure, The Japan Society of Mechanical Engineers, Vol.12, No.11-6 (2011), pp.100-105 (in Japanese).

Okumura, Y., Furuta, T. and Yamanaka, S., Hallucigenia Project: Design Methods for Next-generation Robotic Vehicle, IATSS Rev (IntAssoc Traffic SafSci), Vol.32, No.1 (2007), pp.49-56 (in Japanese).

Sasaki, J., Ota, J., Arai, T., Yoshida, E. and Kurabayashi, D., Cooperative Grasping of an Unknown Object by Multiple Mobile Robots, Journal of Robotics Society of Japan, Vol.14, No.7 (1996), pp.1010-1017 (in Japanese).

Tadakuma, K., Omnidirecitonal mobile and driving mechanism, Journal of Robotics Society of Japan, Vol. 29, No. 6 (2011), pp.516-519 (in Japanese).

Tang, J., Watanabe, K., Kuribayashi, K. and Shiraishi, Y., Autonomous control for an omnidirectional mobile robot with the orthogonal-wheelassembly, Journal of Robotics Society of Japan, Vol.17, No.1 (1999), pp.51-60 (in Japanese).

Tsuji, K. and Murakami, T., Control of load distribution based on environmental information for formation control of multiple mobile robots, The transactions of the Institute of Electrical Engineers of Japan. D, A publication of Industry Applications Society, Vol.124, No.8 (2004), pp.767-773 (in Japanese).

Usuki, Y. and Nakazawa, K., Distributed coordination and environmental load distribution controlfor omni-directional vehicle, Transactions of the Japan Society of Mechanical Engineers, Vol.80, No.817 (2014), Page.WEB ONLY DR0263. (in Japanese)

Wada, M., Modeling and Control of Omnidirectional Mobile Robots with Active Casters, Journal of Robotics Society of Japan, Vol.25, No.7 (2007), pp.1100-1107 (in Japanese).

Yoshimura, M. and Fujimoto, H., Driving Torque Control Method for Electric Vehicle with In-Wheel Motors, IEEJ Transaction on Industry Applications, Vol.131, No.5 (2011), pp.721-728. (in Japanese) 